Supporting information

\title{
Novel Mechanistic Insights into Bacterial Fluoroquinolone Resistance
}

Gao-Fei Du' ${ }^{1}$, Yun-Dan Zheng ${ }^{1}$, Jing Chen ${ }^{2}$, Qing-Yu He ${ }^{1, *}$ and Xuesong Sun ${ }^{1, *}$

${ }^{1}$ Key Laboratory of Functional Protein Research of Guangdong Higher Education Institutes, Institute of Life and Health Engineering, College of Life Science and Technology, Jinan University, Guangzhou 510632, China ;

${ }^{2}$ Department of Clinical Laboratory, Nanfang Hospital, Southern Medical University, Guangzhou 510632, China

Keywords: Evolution; Resistance; Ciprofloxacin

*Correspondence should be addressed to: Prof. Xue-song Sun, Tel: 86-20-85226165, E-mail: tsunxs@jnu.edu.cn; Prof. Qing-Yu He, Tel \& Fax: +86-20-85227039, E-mail: tqyhe@email.jnu.edu.cn 
Table S1. Identification of differentially expressed proteins in $5^{\text {th }}, 28^{\text {th }}$, and $45^{\text {th }}$ round bacterial cultures.

Table S2. Description of clinical Escherichia coli isolates used in this study.

Table S3. Mutation patterns of quinolone-resistance-determining regions in isolates.

Figure S1. Bioinformatic analysis of differentially expressed proteins. (A) Distribution statistics of fold changes in differentially expressed proteins (DEPs) at different antibiotic resistance levels. (B) Visualization of KEGG metabolic pathways of all DEPs and metabolic pathways. (C) Regulatory pathways.

Figure S2. Changes in pathways involved in pentose phosphate and one carbon folate metabolism in bacteria with medium resistance.

Figure S3. Pathway changes involved in pentose phosphate and one carbon pool folate metabolism in highly resistant bacteria.

Figure S4. Cell mobility. (A) Real-time tracking of different generations (replicate1). (B) Levels of cell motility at different resistance levels.

Figure S5. Correlations among different resistance levels. (A) Venn diagram for the number of differentially expressed proteins (DEPs) at different resistance levels. (B) Hierarchical cluster analysis for the intersection of DEPs at identified at these three resistance levels. (C) KEGG pathway enrichment analysis for DEPs at least identified at two resistance levels. (D) KEGG pathway enrichment analysis for DEPs only identified at the high resistance level.

Figure S6. (A) Expression changes in differentially expressed proteins at different resistance levels in DNA replication and repair; white: no significant change, red: up- 
regulated proteins, blue: down-regulated proteins. (B) Relative minimum inhibitory concentration of sulfamethoxazole/trimethoprim (19:1) at different resistance levels. Error bars indicate SD. ${ }^{* * *}, p<0.001$. 

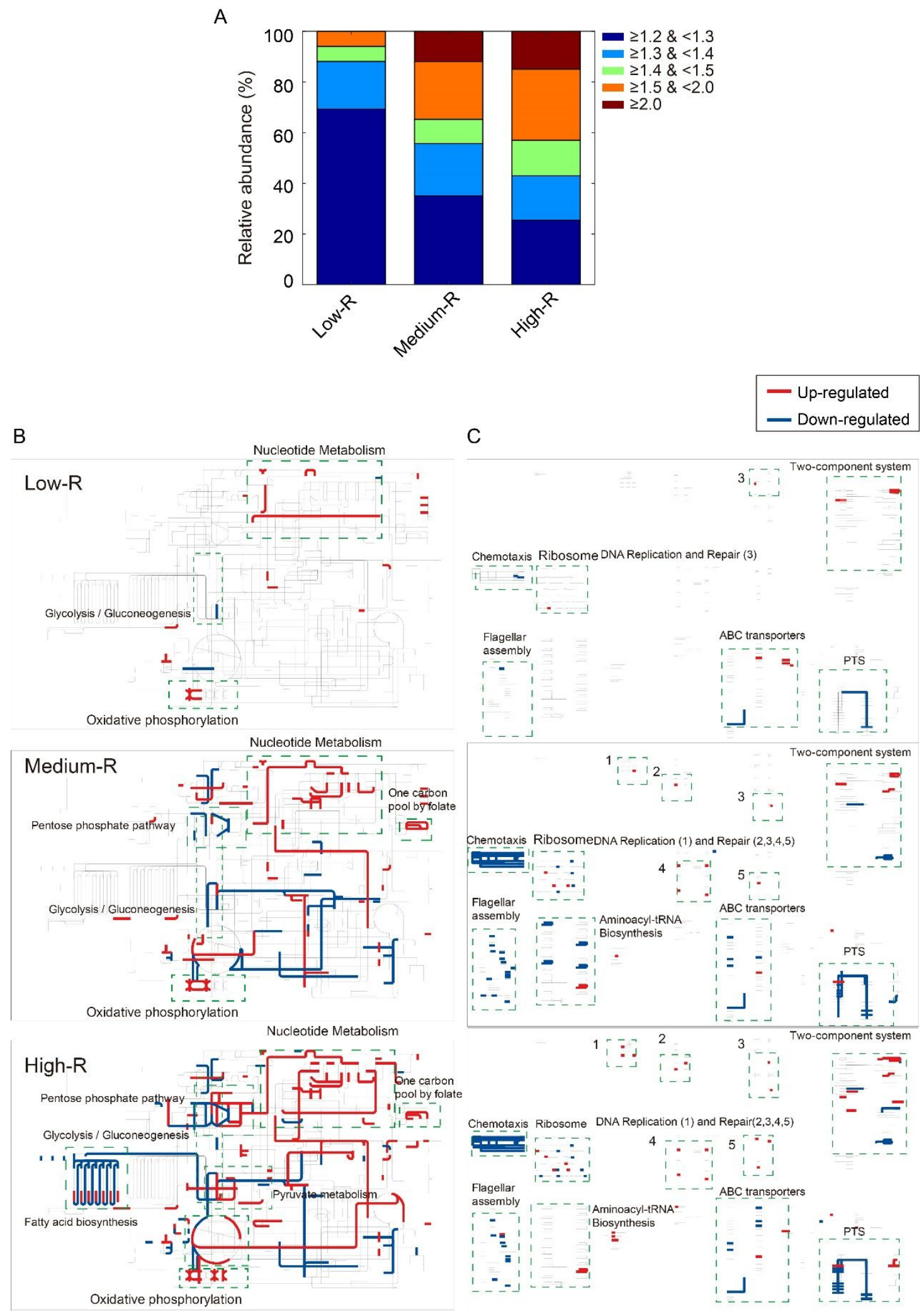

Figure S1. (A) Distribution statistics of fold changes in differentially expressed proteins (DEPs) at different antibiotic resistance levels. (B) Visualization of KEGG metabolic pathways of all DEPs and metabolic pathways. (C) Regulatory pathways. 


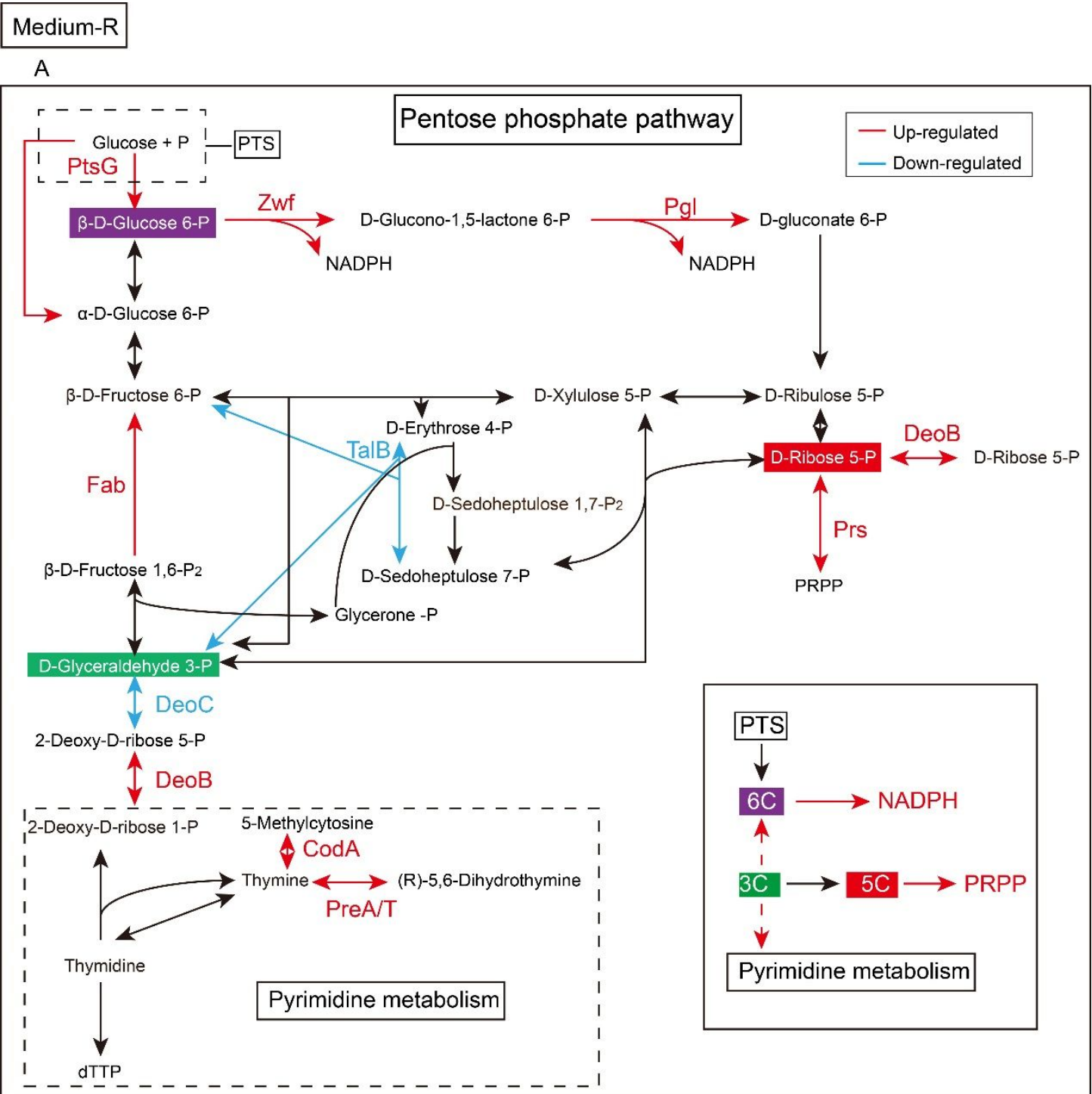

B

One carbon pool by folate

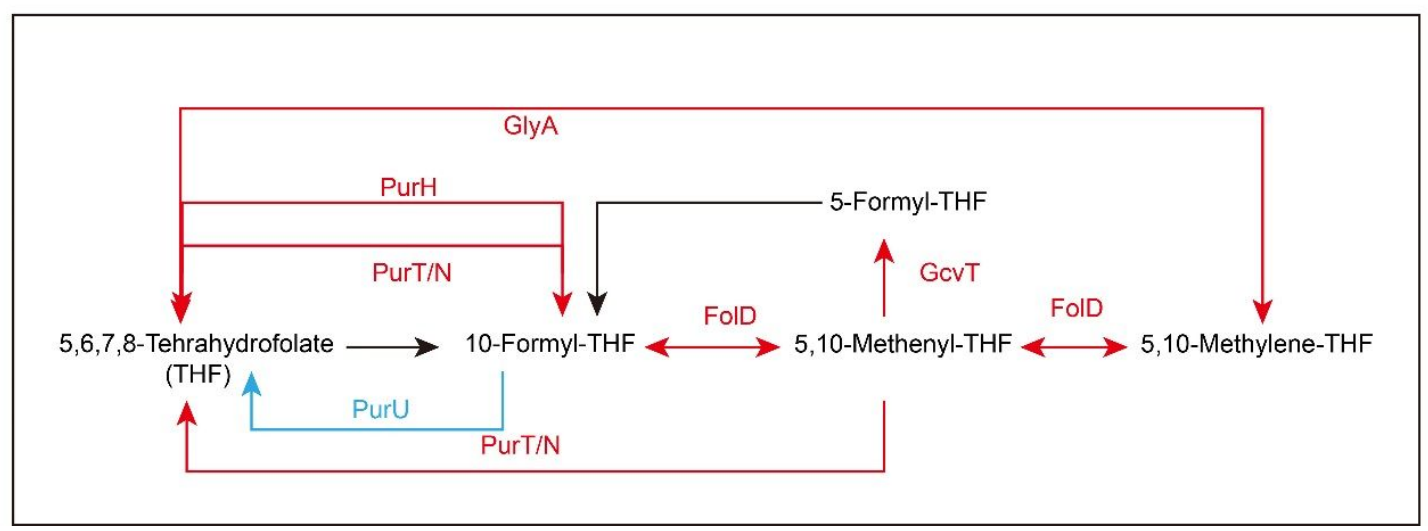

Figure S2. Changes in pathways involved in pentose phosphate and one carbon folate metabolism in bacteria with medium resistance. 
High-R

A

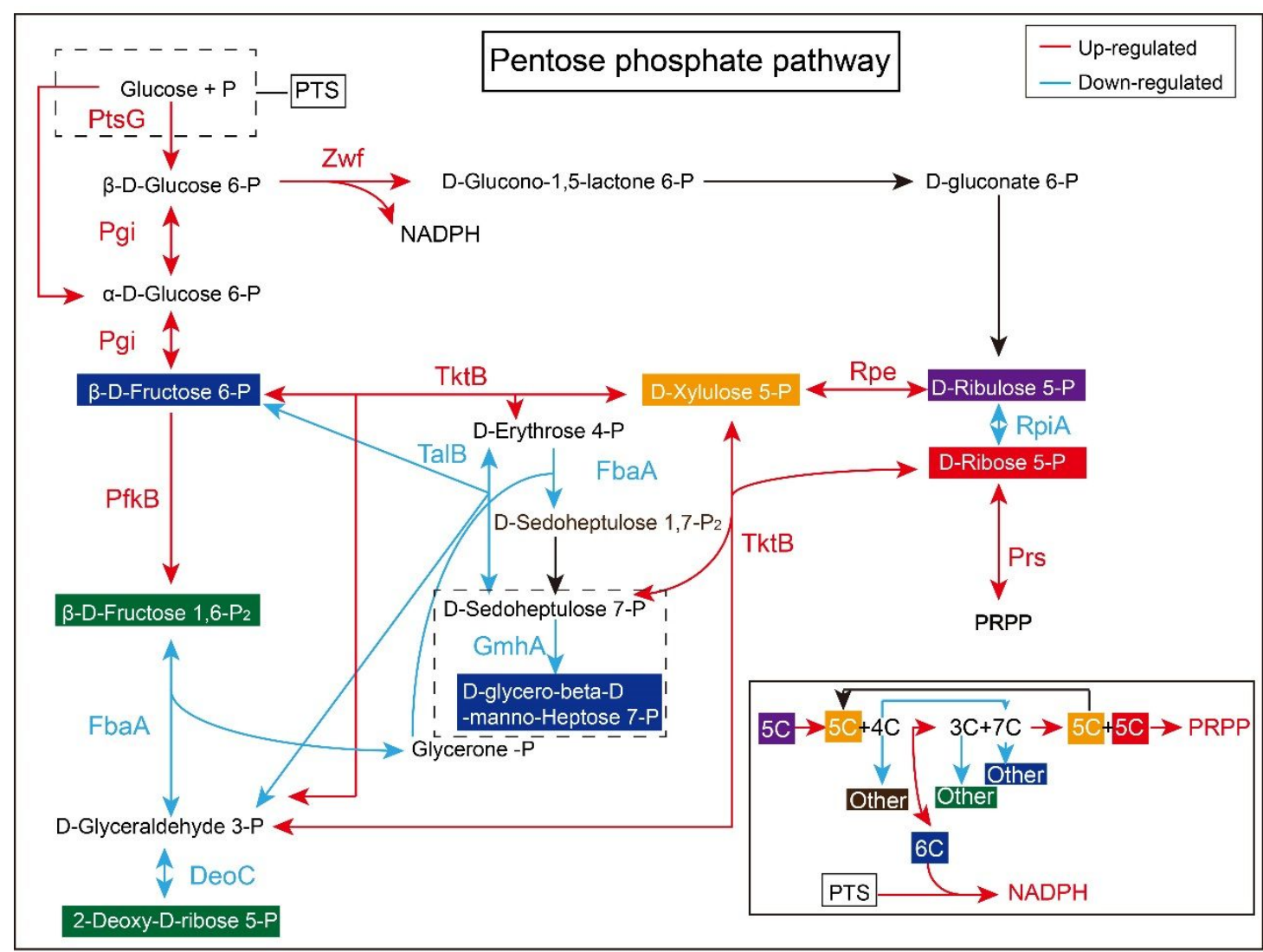

B One carbon pool by folate

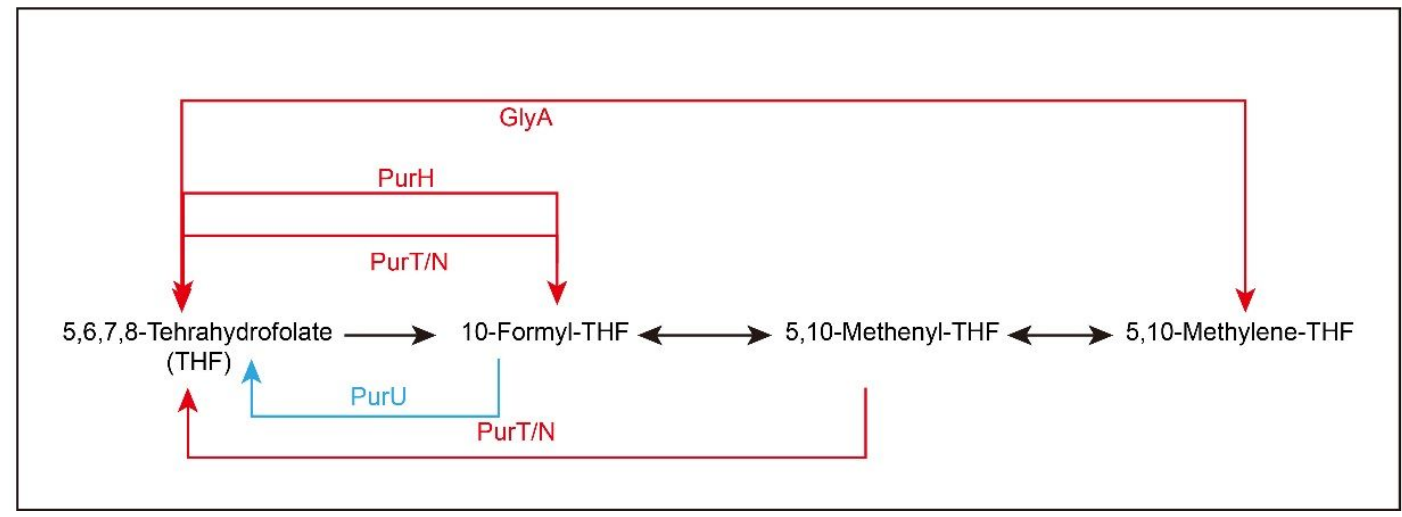

Figure S3. Pathway changes involved in pentose phosphate and one carbon pool folate metabolism in highly resistant bacteria. 
A

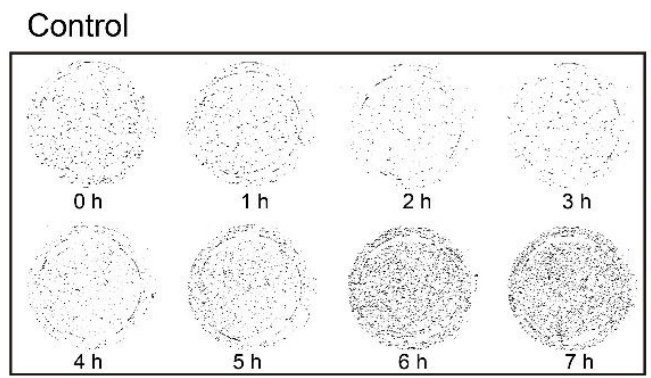

Medium-R

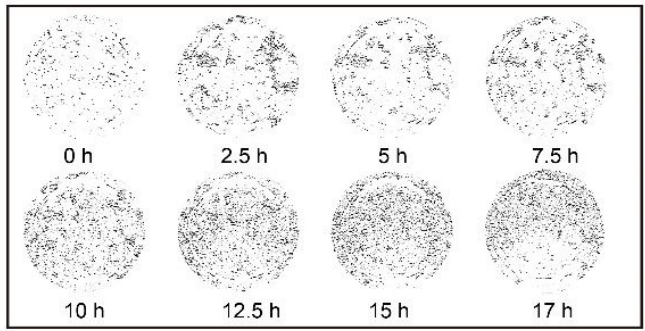

Low-R

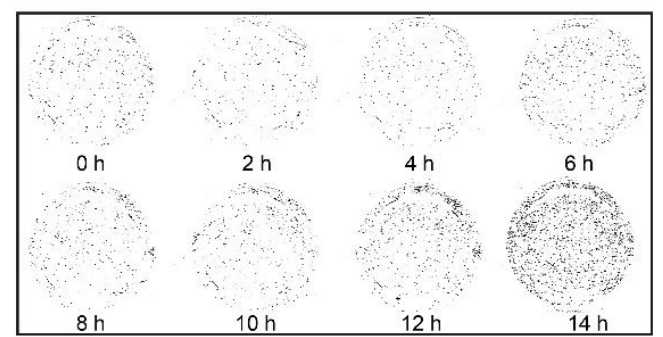

High-R

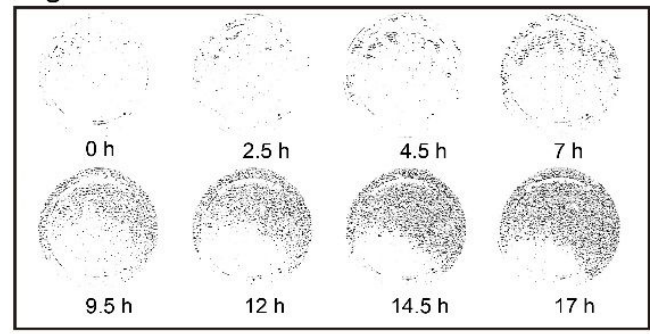

B

Control

Medium-R

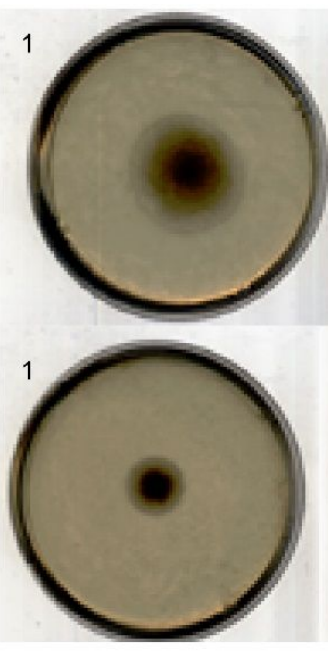

Low-R

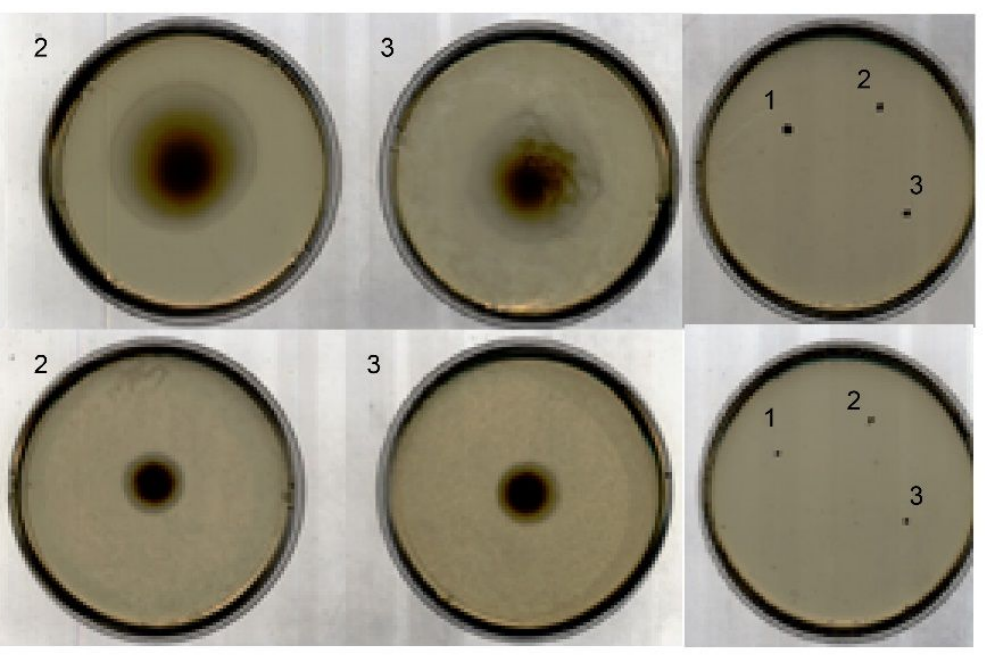

High-R

Figure S4. (A) Real-time tracking of different generations (replicate1). (B) Levels of cell motility at different resistance levels. 
A
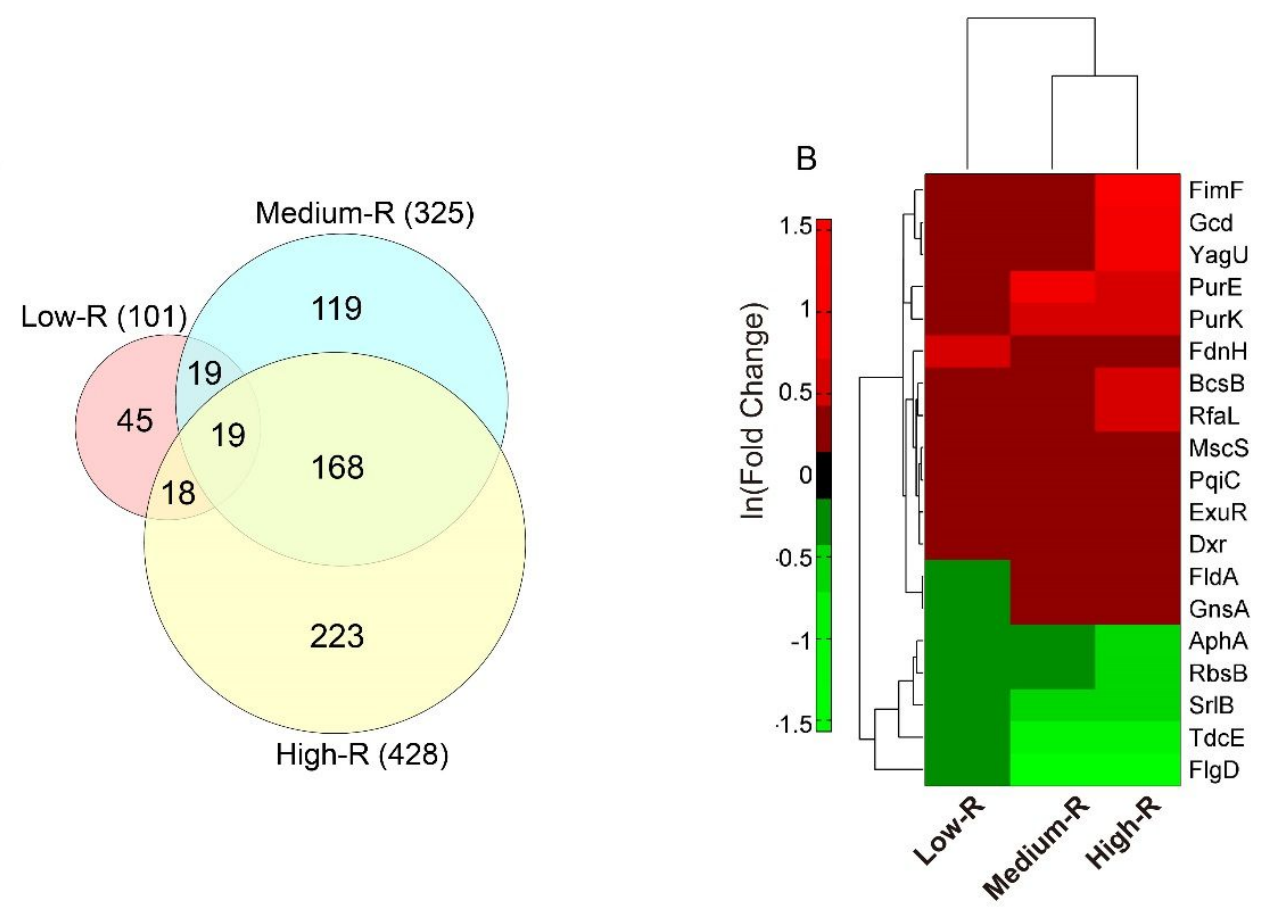

$-\log 10(P$ value $)$

C

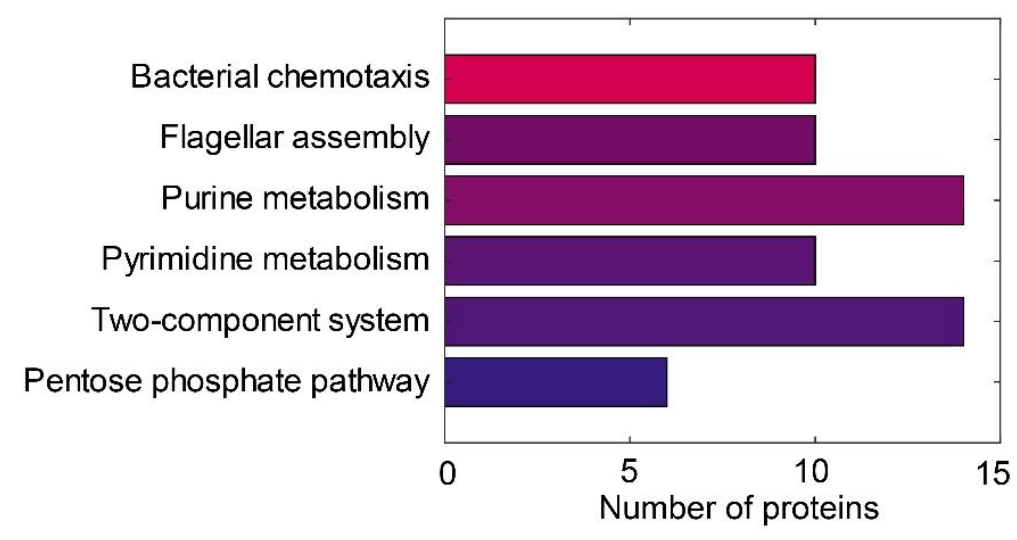

$-\log 10(P$ value $)$

Citrate cycle (TCA cycle)

Amino sugar and nucleotide sugar metabolism

Pyruvate metabolism

Glycolysis / Gluconeogenesis

Pentose phosphate pathway

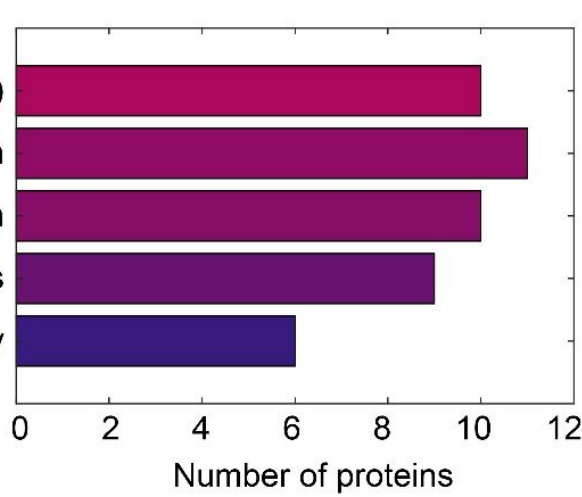

Figure S5. Correlations among different resistance levels. (A) Venn diagram for the number of differentially expressed proteins (DEPs) at different resistance levels. (B) Hierarchical cluster analysis for the intersection of DEPs at identified at these three resistance levels. (C) KEGG pathway enrichment analysis for DEPs at least identified at two resistance levels. (D) KEGG pathway enrichment analysis for DEPs only identified at the high resistance level. 
A

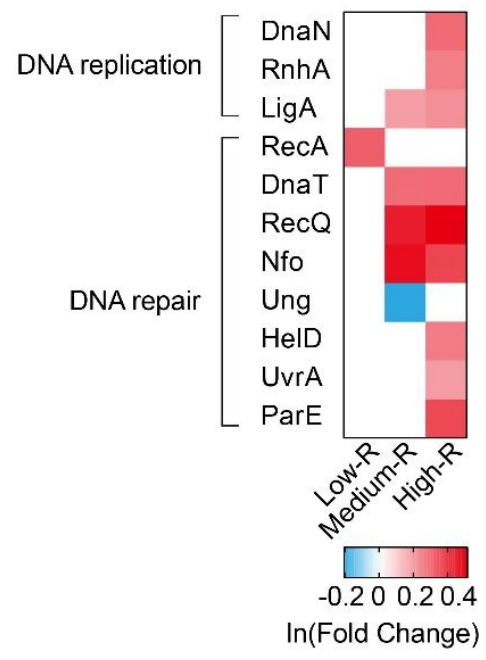

B

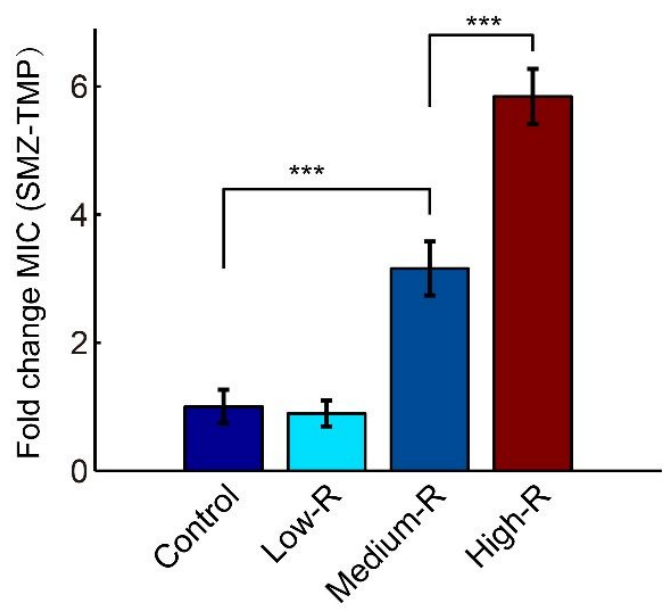

Figure S6. (A) Expression changes in differentially expressed proteins at different resistance levels in DNA replication and repair; white: no significant change, red: upregulated proteins, blue: down-regulated proteins. (B) Relative minimum inhibitory concentration of sulfamethoxazole/trimethoprim (19:1) at different resistance levels. Error bars indicate SD. ${ }^{* * *}, p<0.001$. 